\title{
Age of asteroid sample return
}

\author{
HISAYOSHI YURIMOTO ${ }^{1,2}$ \\ ${ }^{1}$ Natural History Sciences, Hokkaido University, Sapporo \\ 001-0021, Japan \\ ${ }^{2}$ ISAS/JAXA, Sagamihara, Kanagawa 252-5210, Japan
}

Since the final Moon sample return by Apollo 17 in 1972, Hayabusa succeeded the first sample return from an asteroid 25143 Itokawa in 2010 by touchdown on the asteroid. Pickup operation of Itokawa particles larger than $10 \mu \mathrm{m}$ in length from the sample container of Hayabusa started from 2010 and continues today. Itokawa particles listed for international science research exceed 880 grains which sizes are $35 \mu \mathrm{m}$ in length for average and $270 \mu \mathrm{m}$ for maximum. Now, two new asteroid sample return missions, Hayabusa 2 and OSIRISREx, are in progress. Hayabusa 2 succeeded touchdown on an asteroid 162173 Ryugu two times in 2019 to collect samples. Hayabusa 2 will return the samples to Earth in late 2020. OSIRIS-REx reached the proximity of an asteroid 101955 Bennu in 2018 and a contact with the surface is planned in 2020. Conditions of both spacecrafts are healthy.

Chemical and oxygen isotopic compositions of Itokawa particles demonstrate that ordinary chondrites come from Stype asteroids such as Itokawa. Differences of reflected spectrum between ordinary chondrites and S-type asteroids result from effects of space weathering. Analysis of return samples revealed how solar wind irradiation set up space weathering to change chemical and physical properties of mineral surface and how fast the weathering occurred on the asteroid. The space weathering rates reveal dynamics and evolution on the asteroid. These insights had not been approached from meteorite studies because intact surface of meteorites had completely lost by evaporation when meteorites entered the atmosphere.

Unlike Itokawa, Ryugu and Bennu are classified into Ctype and B-type asteroids, respectively, and thought to be corresponding to some kind of carbonaceous chondrites. An albedo of Ryugu is smaller than those of carbonaceous chondrites, but albedo of Bennu is likely to those of carbonaceous chondrites. Water contents of Bennu are moderate, but those of Ryugu are smaller than those of most carbonaceous chondrites. Returned samples will clarify whether Ryugu and Bennu are composed of some kind of carbonaceous chondrites or unique objects unlike known meteorites.

In this talk, I will review materials returned from asteroids and compare them with meteorites. 